\title{
The distributional Henstock-Kurzweil integral and applications II
}

\author{
Wei Liü, Guoju Ye ${ }^{\mathrm{a}}$, Dafang Zhao ${ }^{\mathrm{a}, \mathrm{b}, *}$ \\ ${ }^{a}$ College of Science, Hohai University, Nanjing 210098, P. R. China. \\ ${ }^{b}$ School of Mathematics and Statistics, Hubei Normal University, Huangshi 435002, P. R. China.
}

Communicated by Y. H. Yao

\begin{abstract}
In this paper, we study a special Banach lattice $D_{H K}$, which is induced by the distributional Henstock-Kurzweil integral, and discuss its lattice properties. We show that $\mathrm{D}_{\mathrm{HK}}$ is an AM-space with the Archimedean property and the Dunford-Pettis property but it is not Dedekind complete. We also present two fixed point theorems in $\mathrm{D}_{\mathrm{HK}}$. Meanwhile, two examples are worked out to demonstrate the results. (C)2017 All rights reserved.
\end{abstract}

Keywords: Distributional Henstock-Kurzweil integral, Banach lattice, AM-space, Archimedean property, Dunford-Pettis property, order continuity.

2010 MSC: 46B42, 47H10, 26A42, 46G12.

\section{Introduction}

This is a continuation of the preceding paper [22], where the distributional Henstock-Kurzweil integral and its properties were studied. The distributional Henstock-Kurzweil integral defined by using Schwartz distributional derivative is a very wide integral form. It includes the Henstock-Kurzweil integral and the Lebesgue integral, see details in [12-15, 19, 21, 22]. The space of Henstock-Kurzweil integrable distributions, denoted by $\mathrm{D}_{\mathrm{HK}}$, is a completion of the space of Henstock-Kurzweil integrable functions.

The outline of the present paper is as follows. Section 2 is devoted to the basic notations of the distributional Henstock-Kurzweil integral. In Section 3, an inner product is introduced in the space $\mathrm{D}_{\mathrm{HK}}$ and so $\mathrm{D}_{\mathrm{HK}}$ is an inner product space. Section 4 proves that the space $\mathrm{D}_{\mathrm{HK}}$ is a Banach lattice with a norm cone. Besides, $\mathrm{D}_{\mathrm{HK}}$ is also an AM-space with the Archimedean property and the Dunford-Pettis property, the details are carried out in Section 5. In Section 6, we show that the norm on $\mathrm{D}_{\mathrm{HK}}$ is $\sigma$-order continuous. However, $\mathrm{D}_{\mathrm{HK}}$ is not Dedekind complete. Finally, we end this paper with applications, where two fixed point theorems are presented in $\mathrm{D}_{\mathrm{HK}}$ and two examples are given to demonstrate the results.

\section{Basic definitions and preliminaries}

For convenience, we use the same notations as in [22] and list some basic ones as follows.

Let $(a, b)$ be an open interval in $\mathbb{R}$, we define

\footnotetext{
*Corresponding author

Email addresses: liuw626@hhu.edu.cn (Wei Liu), yegj@hhu.edu.cn (Guoju Ye), dafangzhao@163.com (Dafang Zhao)
} 


$$
\mathcal{D}((a, b))=\left\{\phi:(a, b) \rightarrow \mathbb{R} \mid \phi \in C_{c}^{\infty} \text { and } \phi \text { has a compact support in }(a, b)\right\} .
$$

The distributions on $(a, b)$ are defined to be the continuous linear functionals on $\mathcal{D}((a, b))$. The dual space of $\mathcal{D}((a, b))$ is denoted by $\mathcal{D}^{\prime}((a, b))$.

For all $f \in \mathcal{D}^{\prime}((a, b))$, we define the distributional derivative $f^{\prime}$ of $f$ to be a distribution satisfying $\left\langle f^{\prime}, \phi\right\rangle=-\left\langle f, \phi^{\prime}\right\rangle$, where $\phi \in \mathcal{D}((a, b))$ is a test function. Further, we write distributional derivative as $f^{\prime}$ and its pointwise derivative as $f^{\prime}(t)$ where $t \in \mathbb{R}$. From now on, all derivatives in this paper will be distributional derivatives unless stated otherwise.

Denote the space of continuous functions on $[a, b]$ by $C([a, b])$. Let

$$
\mathrm{C}_{0}=\{\mathrm{F} \in \mathrm{C}([\mathrm{a}, \mathrm{b}]): \mathrm{F}(\mathrm{a})=0\} \text {. }
$$

Then $\mathrm{C}_{0}$ is a Banach space under the norm

$$
\|F\|_{\infty}=\sup _{t \in[a, b]}|F(t)|=\max _{t \in[a, b]}|F(t)| .
$$

Definition 2.1 ([22, Definition 1]). A distribution $f \in \mathcal{D}^{\prime}((a, b))$ is said to be Henstock-Kurzweil integrable (shortly $D_{H K}$ ) on an interval $[a, b]$, if there exists a continuous function $F \in C_{0}$ such that $F^{\prime}=f$, i.e., the distributional derivative of $F$ is $f$. The distributional Henstock-Kurzweil integral of $f$ on $[a, b]$ is denoted by $\int_{a}^{b} f(t) d t=F(b)-F(a)$. The function $F$ is called the primitive of $f$. For short, $\int_{a}^{b} f=F(b)-F(a)$.

For every $f \in D_{H K}, \phi \in \mathcal{D}((a, b))$, we write

$$
\langle f, \phi\rangle=\int_{a}^{b} f(t) \phi(t) d t=-\int_{a}^{b} F(t) \phi^{\prime}(t) d t .
$$

The distributional Henstock-Kurzweil integral is very wide and it includes the integrals of Riemann, Lebesgue, Henstock-Kurzweil, restricted and wide Denjoy (see [14, 21, 22]).

For $f \in D_{H K}$, define the Alexiewicz norm in $D_{H K}$ as

$$
\|f\|=\|F\|_{\infty}=\sup _{t \in[a, b]}|F(t)|=\max _{t \in[a, b]}|F(t)| .
$$

Under the Alexiewicz norm, $\mathrm{D}_{\mathrm{HK}}$ is a Banach space, see [21, Theorem 2]. In [5], the author first proved that the completion, under the Alexiewicz norm, of the family of all Henstock-Kurzweil integrable functions in $[a, b]$, is the space $D_{H K}$.

Let $g:[a, b] \rightarrow \mathbb{R}$, its variation is $V(g)=\sup \sum_{n}\left|g\left(s_{n}\right)-g\left(t_{n}\right)\right|$ where the supremum is taken over every sequence $\left\{\left(t_{n}, s_{n}\right)\right\}$ of disjoint intervals in $[a, b]$. A function $g$ is of bounded variation on $[a, b]$, if $\mathrm{V}(\mathrm{g})$ is finite. Denote the space of functions of bounded variation by $\mathcal{B} \mathcal{V}$. The space $\mathcal{B} \mathcal{V}$ is a Banach space with norm $\|g\|_{\mathcal{B} V}=|g(a)|+V(g)$.

The dual of $D_{H K}$ is $\mathcal{B V}$ (see cf. [21]) and we have

Lemma 2.2 ([21, Theorem 7]). (Hölder inequality) Let $f \in \mathrm{D}_{\mathrm{HK}}$. If $\mathrm{g} \in \mathcal{B} \mathcal{V}$, then

$$
\left|\int_{a}^{b} f g\right| \leqslant 2\|f\|\|g\|_{\mathcal{B} \mathcal{~} \text {. }}
$$

\section{An inner product in $D_{H K}$}

In this section we introduce an inner product in $\mathrm{D}_{\mathrm{HK}}$ so that it is an inner product space.

Let $f, g \in D_{H K}$ with the primitives $F, G \in C_{0}$, respectively. We say that $f=g$ if $F(t)=G(t)$ everywhere.

Define

$$
\langle f, g\rangle=\langle F, G\rangle=\int_{a}^{b} F(t) G(t) d t
$$

Now, we prove that (3.1) is an inner product in $\mathrm{D}_{\mathrm{HK}}$. 
(i) For any $f \in D_{H K}$,

$$
\langle f, f\rangle=\langle F, F\rangle=\int_{a}^{b} F^{2}(t) d t \geqslant 0,
$$

and $\langle f, f\rangle=0$ if and only if $F(t)=0$ almost everywhere, i.e., $f=0$.

(ii) For any $f, g \in D_{H K}$,

(iii) For any $f, g, h \in D_{H K}$,

$$
\langle f, g\rangle=\int_{a}^{b} F(t) G(t) d t=\int_{a}^{b} G(t) F(t) d t=\langle g, f\rangle .
$$

$$
\begin{aligned}
\langle f, g+h\rangle & =\int_{a}^{b} F(t)(G(t)+H(t)) d t \\
& =\int_{a}^{b} F(t) G(t) d t+\int_{a}^{b} F(t) H(t) d t=\langle f, g\rangle+\langle f, h\rangle .
\end{aligned}
$$

By (i), (ii) and (iii), we obtain:

Theorem 3.1. The space $\mathrm{D}_{\mathrm{HK}}$ is an inner product space with the inner product given in (3.1).

The inner product (3.1) induces a norm

$$
\|f\|_{\langle,\rangle}=\left(\int_{a}^{b} F^{2}(t) d t\right)^{\frac{1}{2}} .
$$

It is easy to obtain

$$
\|\mathbf{f}\|_{\langle,\rangle} \leqslant(\mathbf{b}-\mathbf{a})^{\frac{1}{2}}\|\mathbf{f}\| .
$$

This means that the norm $\|\cdot\|$ is stronger than $\|\cdot\|_{\langle,\rangle}$. However, the two norms $\|\cdot\|_{\langle,\rangle}$and $\|\cdot\|$ in $\mathrm{D}_{\mathrm{HK}}$ are not equivalent, because $\mathrm{D}_{\mathrm{HK}}$ is complete under the norm $\|\cdot\|$ but not complete under the norm $\|\cdot\|_{\langle,\rangle}$.

Remark 3.2. The norm $\|\cdot\|$ on $\mathrm{D}_{\mathrm{HK}}$ does not induce an inner product, since $\|\cdot\|$ does not satisfy the parallelogram law.

Remark 3.3. Although $\mathrm{D}_{\mathrm{HK}}$ is an inner product space, it is not complete under the norm $\|\cdot\|_{\langle,\rangle}$. That is, $\mathrm{D}_{\mathrm{HK}}$ is not a Hilbert space under the norm $\|\cdot\|_{\langle,\rangle}$. We know that the Hilbert space is self-conjugate. Since the dual of $\mathrm{D}_{\mathrm{HK}}$ is $\mathcal{B V}, \mathrm{D}_{\mathrm{HK}}$ is not self-conjugate and therefore $\mathrm{D}_{\mathrm{HK}}$ is not a Hilbert space.

\section{The ordering in $\mathrm{D}_{\mathrm{HK}}$ and Banach lattice}

We shall first present some basic properties of order Banach space.

A closed subset $X_{+}$of a Banach space $X$ is called an order cone, if $X_{+}+X_{+} \subseteq X_{+}, X_{+} \cap\left(-X_{+}\right)=\{0\}$ and $c X_{+} \subseteq X_{+}$for each $c \geqslant 0$. It is easy to see that the order relation $\preceq$ defined by

$$
x \preceq y \text {, if and only if } y-x \in X_{+},
$$

is a partial ordering in $X$, and that $X_{+}=\{y \in X \mid 0 \preceq y\}$ is an order cone in $X$. The space $X$, equipped with this partial ordering, is called an ordered Banach space. For any $r>0, B_{r}=\{x \in X:\|x\| \leqslant r\}$ is called a closed ball in $X$. The order interval $[y, z]=\{x \in X \mid y \preceq x \preceq z\}$ is a closed subset of $X$ for all $y, z \in X$. A sequence (subset) of $X$ is called order bounded, if it is contained in an order interval $[y, z]$ of $X$. We say that an order cone $X_{+}$of a Banach space is normal, if there exists a constant $\gamma \geqslant 1$ such that

$$
0 \preceq x \preceq y \text { in } X \text { implies }\|x\| \leqslant \gamma\|y\| .
$$

$\mathrm{X}_{+}$is called regular, if all increasing and order bounded sequences of $\mathrm{X}_{+}$converge. If all normbounded and increasing sequences of $X_{+}$converge, we say that $X_{+}$is fully regular. As for the proof of the following result, see, e.g., [11, Theorem 2.2.2]. 
Lemma 4.1. Let $\mathrm{X}_{+}$be an order cone of a Banach space $\mathrm{X}$. If $\mathrm{X}_{+}$is fully regular, it is also regular, and if $\mathrm{X}_{+}$is regular, it is also normal. Converse holds if $\mathrm{X}$ is reflexive.

Assume that $X$ is an order linear space. If for every $x, y \in X$, there exists $z \in X$ such that $x \preceq z, y \preceq z$, and if $x \preceq \mathfrak{u}, y \preceq u$ then $z \preceq u$, then $X$ is called a Riesz space (or lattice) and we denote $z=x \vee y$.

A vector subspace $M$ of a Riesz space $X$ is said to be a Riesz subspace (or a vector sublattice), whenever $M$ is closed under the lattice operations of $X$, i.e., whenever for each pair $x, y \in M$ the vector $x \vee y$ (taken in $X$ ) belongs to $M$.

For a vector $x$ in a lattice $X$, define $x^{+}=x \vee 0, x^{-}=(-\chi) \vee 0$ and $|x|=x \vee(-x)$, then we call them the positive part, the negative part and the absolute value (or modulus) respectively. Moreover, $x=x^{+}-x^{-}$ and $|x|=x^{+}+x^{-}$. Note that $|x|=0$, if and only if $x=0$.

Definition 4.2. Assume that $X$ is a Banach space, if $X$ is a lattice and

$$
|x| \preceq|y| \text { in } X \text { implies }\|x\| \leqslant\|y\|,
$$

then $X$ is called a Banach lattice and the norm $\|\cdot\|$ satisfying (4.1) is called a lattice norm.

Recall that $C([a, b])$ is a Banach lattice with the uniform norm and so is $C_{0}([a, b])$. For $F \in C_{0}([a, b])$, the positive part $\mathrm{F}^{+}=\mathrm{F} \vee 0=\max _{\mathrm{t} \in[\mathrm{a}, \mathrm{b}]}\{\mathrm{F}(\mathrm{t}), 0\}$, the negative part $\mathrm{F}^{-}=(-\mathrm{F}) \vee 0=\max _{\mathrm{t} \in[\mathrm{a}, \mathrm{b}]}\{-\mathrm{F}(\mathrm{t}), 0\}$, and hence $F=F^{+}-F^{-}$and the absolute value $|F|=F^{+}+F^{-}$. Moreover, $F^{+}, F^{-},|F|$ all belong to $C_{0}([a, b])$.

Let $f \in D_{H K}$ with the primitive $F \in C_{0}([a, b])$, define

$$
f^{+}=\left(F^{+}\right)^{\prime}, \quad f^{-}=\left(F^{-}\right)^{\prime}, \quad|f|=|F|^{\prime} .
$$

Then,

$$
f=f^{+}-f^{-}, \quad|f|=f^{+}+f^{-} .
$$

See details in [21].

In $C_{0}([a, b])$ there exists a pointwise order: for $F, G \in C_{0}([a, b]), F \leqslant G$, if and only if $F(t) \leqslant G(t)$, for all $t \in[a, b]$. For $f, g \in D_{H K}$ with primitives $F, G \in C_{0}([a, b])$, respectively, let

$$
f \preceq g \text { (or } g \succeq f) \text {, if and only if } F \leqslant G .
$$

Theorem 4.3 ([21, Theorem 23]). $\mathrm{D}_{\mathrm{HK}}$ is a Banach lattice.

In the Banach lattice $D_{H K}$, define

$$
\mathrm{D}_{\mathrm{HK}+}=\left\{\mathrm{f} \in \mathrm{D}_{\mathrm{HK}}: \mathrm{f} \succeq 0\right\} .
$$

Then $\mathrm{D}_{\mathrm{HK}+}$ is an order cone. Moreover, one has

$$
\begin{aligned}
0 \preceq \mathrm{f} \preceq \mathrm{g} & \Rightarrow 0 \leqslant \mathrm{~F} \leqslant \mathrm{G} \Rightarrow 0 \leqslant \mathrm{~F}(\mathrm{t}) \leqslant \mathrm{G}(\mathrm{t}) \Rightarrow\|\mathrm{F}\|_{\infty} \leqslant\|\mathrm{G}\|_{\infty} \\
& \Rightarrow\|\mathrm{f}\| \leqslant\|\mathrm{g}\| .
\end{aligned}
$$

Therefore, the following statement holds.

Theorem 4.4. $\mathrm{D}_{\mathrm{HK}+}$ is a normal cone in $\mathrm{D}_{\mathrm{HK}}$.

Remark 4.5. In [22], another ordering was introduced in $\mathrm{D}_{\mathrm{HK}}$ and the cone $\mathrm{D}_{\mathrm{HK}+}$ there is proved to be regular. However, in Section 6, we will prove that the cone $\mathrm{D}_{\mathrm{HK}+}$ in (4.3) is not regular, still less full regular.

\section{AM-space}

This section shows that $D_{H K}$ is an AM-space. Moreover, we prove that $D_{H K}$ possesses the Archimedean property (Theorem 5.7) and the Dunford-Pettis property (Theorem 5.8). 
Definition 5.1. A lattice norm on a Riesz space is:

1. an M-norm, if $x, y \succeq 0$ implies $\|x \vee y\|=\max \{\|x\|,\|y\|\} ;$

2. an L-norm, if $x, y \succeq 0$ implies $\|x+y\|=\|x\|+\|y\|$.

A normed Riesz space equipped with an M-norm (resp. an L-norm) is called an M-space. A norm complete M-space (resp. L-space) is an AM-space (resp. AL-space).

Theorem 5.2. $\mathrm{D}_{\mathrm{HK}}$ is an AM-space.

Proof. Let $f, g \in D_{H K}$ and $f, g \succeq 0$ with the primitives $F$ and $G$. Then $F, G \in C_{0}$ and $F(t) \geqslant 0, G(t) \geqslant 0$ for every $t \in[a, b]$. Therefore,

$$
\begin{aligned}
\|\mathrm{f} \vee \mathrm{g}\| & =\|\mathrm{F} \vee \mathrm{G}\|_{\infty}=\max _{\mathrm{t}}\{\mathrm{F}(\mathrm{t}), \mathrm{G}(\mathrm{t})\}=\max \left\{\|\mathrm{F}\|_{\infty},\|\mathrm{G}\|_{\infty}\right\} \\
& =\max \{\|\mathrm{f}\|,\|\mathrm{g}\|\} .
\end{aligned}
$$

This means that Alexiewicz norm $\|\cdot\|$ in $D_{H K}$ is M-norm. Note that $D_{H K}$ is complete, hence $D_{H K}$ is an AM-space.

Lemma 5.3 ([1, Theorem 9.27]). A Banach lattice is an AL-space (resp. an AM-space), if and only if its dual is an AM-space (resp. an AL-space).

Theorem 5.4. $\mathcal{B} \mathcal{V}$ is an $A L$-space.

Proof. By Theorem 5.2, $\mathrm{D}_{\mathrm{HK}}$ is AM-space. It follows from Lemma 5.3 that $\mathcal{B} \mathcal{V}$ is an AL-space, because $\mathcal{B} \mathcal{V}$ is the dual of $D_{H K}$.

A vector $e>0$ in a Riesz space $X$ is an order unit, or simply a unit, if for each $x \in X$ there exists some $\lambda>0$ such that $|x| \preceq \lambda e$. It is easy to see that $D_{H K}$ is a Banach lattice with unit.

Two Riesz spaces $X$ and $Y$ are lattice isomorphic, (or Riesz isomorphic or simply isomorphic), if there exists a one-to-one, onto, lattice preserving linear operator $T: X \rightarrow Y$. That is, besides being linear, one-to-one, and surjective, $\mathrm{T}$ also satisfies the identities

$$
T(x \vee y)=T(x) \vee T(y) \text { and } T(x \wedge y)=T(x) \wedge T(y),
$$

for all $x, y \in X$.

The Kakutani-Bohnenblust-M.Krein-S.Krein theorem ([1, Theorem 9.32]) shows that a Banach lattice is an AM-space with unit, if and only if it is lattice isometric to $\mathrm{C}(\mathrm{K})$ for some compact Hausdorff space $\mathrm{K}$. The space $\mathrm{K}$ is unique (up to homeomorphism). So, we have the following result.

Theorem 5.5. Banach lattice $\mathrm{D}_{\mathrm{HK}}$ is lattice isometric to $\mathrm{C}([\mathrm{a}, \mathrm{b}])$.

Proof. The proof follows from Theorem 5.2 and the Kakutani-Bohnenblust-M.Krein-S.Krein theorem.

Now, we consider the Archimedean property and the Dunford-Pettis Property of $\mathrm{D}_{\mathrm{HK}}$.

Recall that a net $\left\{x_{\alpha}\right\}$ in a Riesz space is decreasing, written $x_{\alpha} \downarrow$, if $\alpha \geqslant \beta$ implies $x_{\alpha} \preceq x_{\beta}$. The symbol $x_{\alpha} \uparrow$ indicates an increasing net, while $x_{\alpha} \uparrow \preceq x$ (resp. $\left.x_{\alpha} \downarrow \succeq x\right)$ denotes an increasing (resp. decreasing) net that is order bounded from above (resp. below) by $x$. The notation $x_{\alpha} \downarrow x$ means that $x_{\alpha} \downarrow$ and $\inf \left\{x_{\alpha}\right\}=x$. The meaning of $x_{\alpha} \uparrow x$ is similar.

Definition 5.6. A Riesz space $X$ is Archimedean, whenever $\frac{1}{n} \chi \downarrow 0$ holds in $X$ for each $x \in X^{+}$.

Theorem 5.7. $\mathrm{D}_{\mathrm{HK}}$ has the Archimedean property.

Proof. Suppose that $f \in D_{H K}$ with the primitive $F \in C_{0}([a, b])$ and $0 \preceq f$ on $[a, b]$. Then $0 \leqslant F(t)$ for each $t \in[a, b]$. So, $\frac{1}{n} F(t) \downarrow 0$ in $\mathbb{R}$ for each $t$. Hence, by the Dini theorem, $\frac{1}{n} F \downarrow 0$ uniformly. It follows that $\frac{1}{n} \mathrm{f} \downarrow 0$ in $D_{H K}$. By Definition 5.6, $D_{H K}$ has the Archimedean property and the proof is complete. 
A Banach space $X$ has the Dunford-Pettis Property, if $x_{n} \stackrel{w}{\rightarrow} x$ in $X$ and $x_{n}^{\prime} \stackrel{w}{\rightarrow} x^{\prime}$ in $X^{\prime}$ imply $\left\langle x_{n}^{\prime}, x_{n}\right\rangle \rightarrow$ $\left\langle x^{\prime}, x\right\rangle$, where $\stackrel{\text { w }}{\longrightarrow}$ " stands for the weak convergence, see more details in $[2,3]$.

Theorem 5.8. $\mathrm{D}_{\mathrm{HK}}$ and $\mathcal{B} \mathcal{V}$ possesses the Dunford-Pettis Property.

Proof. The Grothendieck theorem ([1, Theorem 9.37]) shows that an AM-space (or AL-space) possesses the Dunford-Pettis Property. Since $\mathrm{D}_{\mathrm{HK}}$ is an AM-space and $\mathcal{B V}$ is an AL-space, the assertion follows immediately.

\section{The $\sigma$-order continuity}

In this section, we show that the norm on $D_{H K}$ is $\sigma$-order continuous but $D_{H K}$ is not Dedekind complete.

Definition 6.1 ([1]). A lattice norm $\|\cdot\|$ on a Riesz space is

(a) order continuous, if $x_{\alpha} \downarrow 0$ implies $\left\|x_{\alpha}\right\| \downarrow 0$.

(b) $\sigma$-order continuous, if $x_{n} \downarrow 0$ implies $\left\|x_{n}\right\| \downarrow 0$.

Obviously, order continuity implies $\sigma$-order continuity. The converse is false, even for Banach lattices.

Theorem 6.2. The norm $\|\cdot\|$ on $\mathrm{D}_{\mathrm{HK}}$ defined as in (4.2) is $\sigma$-order continuous.

Proof. Suppose that $f_{n} \in D_{H K}$ with the primitive $F_{n}, n=1,2, \cdots$, and $f_{n} \downarrow 0$. Then $F_{n}(t) \downarrow 0$ for each $t \in[a, b]$. By the Dini Theorem, $\left\{F_{n}\right\}$ uniformly converges to 0 . It implies $\left\|F_{n}\right\|_{\infty} \downarrow 0$ in $C([a, b])$ and therefore $\left\|f_{n}\right\| \downarrow 0$ in $D_{H K}$. So, the norm $\|\cdot\|$ on the $D_{H K}$ is $\sigma$-order continuous and the proof is complete.

A Riesz space $X$ is order complete, or Dedekind complete, if every nonempty subset that is order bounded from above has a supremum. (Equivalently, if every nonempty subset that is bounded from below has an infimum).

Assume that $X$ is a Banach lattice, if for any upper bounded sequence $\left\{x_{n}\right\}$ has supremum $\vee_{n} x_{n}$, then $X$ is called $\sigma$-complete (or $K_{\sigma}$-space). If for any upper bounded set has supremum, then $X$ is called $\mathrm{K}$-space. Obviously, $\mathrm{K}$-space implies $\mathrm{K}_{\sigma}$-space. The converse is false.

It is a pity that in $\mathrm{D}_{\mathrm{HK}}$ the monotone convergence theorem is not true and so $\mathrm{D}_{\mathrm{HK}}$ is not an $\mathrm{K}_{\sigma}$-space, although $\mathrm{D}_{\mathrm{HK}}$ is a Banach lattice.

In fact, in $C_{0}([a, b])$ there exists $\left\{F_{n}\right\}$ such that $-1 \leqslant F_{n} \uparrow \leqslant 0$ in $C_{0}[a, b]$, where 0 is the zero function, but $\left\{F_{n}\right\}$ does not have a supremum in $C_{0}([a, b])$. For example,

$$
F_{n}(t)= \begin{cases}0, & \text { if } 0 \leqslant t \leqslant \frac{1}{2}-\frac{1}{n} \\ -n\left(t-\frac{1}{2}\right)-1, & \text { if } \frac{1}{2}-\frac{1}{n}<t<\frac{1}{2} \\ -1, & \text { if } \frac{1}{2} \leqslant t \leqslant 1\end{cases}
$$

The limit function of $F_{n}$ is

$$
F(t)= \begin{cases}0, & \text { if } 0 \leqslant t<\frac{1}{2} \\ -1, & \text { if } \frac{1}{2} \leqslant t \leqslant 1\end{cases}
$$

which is not in $C_{0}([0,1])$.

By (6.1) and (6.2), it is easy to verify that $D_{H K}$ is not order complete, that is,

Theorem 6.3. $\mathrm{D}_{\mathrm{HK}}$ is not $\mathrm{K}_{\sigma}$-space, and also not $\mathrm{K}$-space.

According to Theorem 6.3, we obtain the following consequence.

Corollary 6.4. The cone $\mathrm{D}_{\mathrm{HK}+}$ is not regular and so is not full regular. 
However, $\mathrm{D}_{\mathrm{HK}}$ can have Dedekind completions as $\hat{\mathrm{D}}_{\mathrm{HK}}$, since, by [1, Theorem 8.8], every Archimedean Riesz space has a unique (up to lattice isomorphism) Dedekind completion. That is, the Dedekind completion of $D_{H K}$ is an order complete Riesz space $\hat{D}_{H K}$ having a Riesz subspace $M$ that is lattice isomorphic to $D_{H K}$ (hence $M$ can be identified with $D_{H K}$ ) satisfying

$$
\hat{f}=\sup \{f \in M: f \preceq \hat{f}\}=\inf \{g \in M: \hat{f} \preceq g\},
$$

for each $\hat{f} \in \hat{D}_{H K}$.

\section{Fixed point theorems and applications}

In this section, we apply the conclusions in Section 5 to establish fixed point theorems in $\mathrm{D}_{\mathrm{HK}}$. The obtained results are used to prove the existence of solutions of an operator equation and a Volterra integral equation.

Let $B$ be a subset of an order Banach space $X$. An operator $T: B \rightarrow B$ is a nonexpansive operator, if $\|T(x)-T(y)\| \leqslant\|x-y\|, \forall x, y \subset B$.

Lemma 7.1 ([20, Corollary 1]). Suppose $\mathrm{X}$ is an AM-space. If $\mathrm{B}_{\mathrm{r}} \subset \mathrm{X}$ is a closed ball and $\mathrm{T}: \mathrm{B}_{\mathrm{r}} \rightarrow \mathrm{B}_{\mathrm{r}}$ is a nonexpansive operator, then $\mathrm{T}$ has a fixed point in $\mathrm{B}_{\mathrm{r}}$.

Lemma 7.2 ([20, Corollary 2]). Suppose $\mathrm{X}$ is an AM-space. If $\mathrm{I} \subset \mathrm{X}$ is a closed order interval and $\mathrm{T}: \mathrm{I} \rightarrow \mathrm{I}$ is a nonexpansive operator, then $\mathrm{T}$ has a fixed point in $\mathrm{I}$.

According to Theorem 5.2 and Lemmas 5.3, 7.1 and 7.2, it is easy to see the following results.

Theorem 7.3. If $\mathrm{T}: \mathrm{B}_{\mathrm{r}} \rightarrow \mathrm{B}_{\mathrm{r}}$ is a nonexpansive operator, where

$$
\mathrm{B}_{\mathrm{r}}=\left\{x \in \mathrm{D}_{\mathrm{HK}}:\|x\| \leqslant \mathrm{r}\right\} .
$$

Then the operator $\mathrm{T}$ has a fixed point in $\mathrm{B}_{\mathrm{r}}$.

Theorem 7.4. If $\mathrm{T}: \mathrm{I} \rightarrow \mathrm{I}$ is a nonexpansive operator, where

$$
\mathrm{I}=[y, z]=\left\{x \in \mathrm{D}_{\mathrm{HK}}: \mathrm{y} \preceq x \preceq z\right\} .
$$

Then the operator Thas a fixed point in $\mathrm{I}$.

Example 7.5. Consider an operator equation

$$
T x=f(t, x), \quad t \in[0,1],
$$

where $x \in D_{H K}, f:[0,1] \times D_{H K} \rightarrow D_{H K}$. If there exist $y, z \in D_{H K}$ such that

$$
y \preceq f(., x) \preceq z, \quad \forall x \in[y, z],
$$

and

$$
\left\|f\left(., x_{1}\right)-f\left(., x_{2}\right)\right\| \leqslant\left\|x_{1}-x_{2}\right\|, \quad \forall x_{1}, x_{2} \in[y, z] .
$$

Then $T$ has a fixed point in $[y, z]$.

Proof. For each $x \in[y, z]$, one has

$$
y \preceq T x=f(t, x) \preceq z,
$$

i.e., $T([y, z]) \subset[y, z]$. Moreover, for any $x_{1}, x_{2} \in[y, z]$, it is easy to see that

$$
\left\|T x_{1}-T x_{2}\right\|=\left\|f\left(t, x_{1}\right)-f\left(t, x_{2}\right)\right\| \leqslant\left\|x_{1}-x_{2}\right\|,
$$

which implies that $\mathrm{T}$ is a nonexpansive operator. In view of Theorem 7.4, the assertion follows immediately. 
Example 7.6. Consider a Volterra integral equation of the type

$$
x(t)=g(t)+\int_{0}^{t} K(t, s) f(s, x(s)) d s, \quad t \in[0,1],
$$

where $x, g \in D_{H K}, f:[0,1] \times D_{H K} \rightarrow D_{H K}, K:[0,1] \times[0,1] \rightarrow \mathbb{R}$ is a continuous function with bounded variation. If there exist positive constants $r$, $L$ such that

$$
\|g\| \leqslant \frac{r}{2}, \quad\|K\| \leqslant \frac{1}{2 L},
$$

and

$$
\|f(., x)-f(., y)\| \leqslant L\|x-y\|, \quad\|f(., x)\| \leqslant \frac{L}{2}\|x\|, \quad \forall x, y \in B_{r},
$$

where $B_{r}$ is defined as in (7.1). Then, the Volterra integral equation (7.2) has a solution.

Proof. Define an operator $\mathrm{T}: \mathrm{B}_{\mathrm{r}} \rightarrow \mathrm{D}_{\mathrm{HK}}$

$$
T x(t):=g(t)+\int_{0}^{t} K(t, s) f(s, x(s)) d s, \quad t \in[0,1]
$$

From (7.3)-(7.5) and Lemma 2.2, it follows that

$$
\|T x\| \leqslant\|g\|+2\|K\|\|f\| \leqslant \frac{r}{2}+\frac{r}{2}=r,
$$

and

$$
\|T x-T y\| \leqslant 2\|K\|\|f(., x)-f(., y)\| \leqslant\|x-y\| .
$$

Therefore, $T: B_{r} \rightarrow B_{r}$ is a nonexpansive operator. By virtue of Theorem 7.3, $T$ has a fixed point in $B_{r}$, i.e., the Volterra integral equation (7.2) has a solution.

Remark 7.7. In Examples 7.5 and 7.6, we deal with equations involving distributions, e.g., let

$$
f(t, x)=h(x)+\left(\sum_{n=1}^{\infty} \frac{\sin n^{2} \pi t}{n^{2}}\right)^{\prime},
$$

where $h(x)$ is continuous with respect to $x \in C([0,1])$ and $(.)^{\prime}$ denotes the distributional derivative. According to [22, Remark 1], $f(t, x)$ is neither Henstock-Kurzweil integrable nor Lebesgue integrable on $[0,1]$, so approaches in the literatures $[4,6-10,16-18]$ are no longer effective. This implies that our results are more general.

\section{Acknowledgment}

The authors wish to thank the referees for their valuable comments and suggestions. This research was supported by the program of High-end Foreign Experts of the SAFEA (No. GDW 20163200216).

\section{References}

[1] C. D. Aliprantis, K. C. Border, Infinite dimensional analysis, A hitchhiker's guide, Third edition, Springer, Berlin, (2006). 5.3, 5, 5, 6.1, 6

[2] C. D. Aliprantis, O. Burkinshaw, Dunford-Pettis operators on Banach lattices, Trans. Amer. Math. Soc., 274 (1982), 227-238. 5

[3] C. D. Aliprantis, O. Burkinshaw, Positive operators, Pure and Applied Mathematics, Academic Press, Inc., Orlando, FL, (1985). 5 
[4] A. Ben Amar, Some fixed point theorems and existence of weak solutions of Volterra integral equation under HenstockKurzweil-Pettis integrability, Comment. Math. Univ. Carolin., 52 (2011), 177-190. 7.7

[5] B. Bongiorno, Relatively weakly compact sets in the Denjoy space, The collection of theses of Symposium on Real Analysis, Xiamen, (1993), J. Math. Study, 27 (1994), 37-44. 2

[6] D. Bugajewski, On the Volterra integral equation and the Henstock-Kurzweil integral, Math. Pannon., 9 (1998), 141-145. 7.7

[7] T. S. Chew, On Kurzweil generalized ordinary differential equations, J. Differential Equations, 76 (1988), $286-293$.

[8] T. S. Chew, F. Flordeliza, On $x^{\prime}=f(t, x)$ and Henstock-Kurzweil integrals, Differential Integral Equations, 4 (1991), 861-868.

[9] M. Federson, R. Bianconi, Linear integral equations of Volterra concerning Henstock integrals, Real Anal. Exchange, 25 (1999/00), 389-417.

[10] M. Federson, R. Bianconi, Linear Volterra-Stieltjes integral equations in the sense of the Kurzweil-Henstock integral, Arch. Math. (Brno), 37 (2001), 307-328. 7.7

[11] D.-J. Guo, Y. J. Cho, J. Zhu, Partial ordering methods in nonlinear problems, Nova Science Publishers, Inc., Hauppauge, NY, (2004). 4

[12] J. Kurzweil, Henstock-Kurzweil integration: its relation to topological vector spaces, Series in Real Analysis, World Scientific Publishing Co., Inc., River Edge, NJ, (2000). 1

[13] J. Kurzweil, Integration between the Lebesgue integral and the Henstock-Kurzweil integral, Its relation to local convex vector spaces, Series in Real Analysis, World Scientific Publishing Co., Inc., River Edge, NJ, (2002).

[14] P. Y. Lee, Lanzhou lectures on Henstock integration, Series in Real Analysis, World Scientific Publishing Co., Inc., Teaneck, NJ, (1989). 2

[15] P. Y. Lee, R. Výborný, Integral: an easy approach after Kurzweil and Henstock, Australian Mathematical Society Lecture Series, Cambridge University Press, Cambridge, (2000). 1

[16] D. O'Regan, Fixed-point theory for weakly sequentially continuous mappings, Math. Comput. Modelling, 27 (1998), 1-14. 7.7

[17] B. Satco, Volterra integral inclusions via Henstock-Kurzweil-Pettis integral, Discuss. Math. Differ. Incl. Control Optim., 26 (2006), 87-101.

[18] B. R. Satco, Nonlinear Volterra integral equations in Henstock integrability setting, Electron. J. Differential Equations, 2008 (2008), 9 pages. 7.7

[19] S. Schwabik, G.-J. Ye, Topics in Banach space integration, Series in Real Analysis, World Scientific Publishing Co. Pte. Ltd., Hackensack, NJ, (2005). 1

[20] P. M. Soardi, Existence of fixed points of nonexpansive mappings in certain Banach lattices, Proc. Amer. Math. Soc., 73 (1979), 25-29 7.1, 7.2

[21] E. Talvila, The distributional Denjoy integral, Real Anal. Exchange, 33 (2008), 51-82. 1, 2, 2.2, 4, 4.3

[22] G.-J. Ye, W. Liu, The distributional Henstock-Kurzweil integral and applications, Monatsh. Math., 181 (2016), $975-989$. $1,2,2.1,2,4.5,7.7$ 